\title{
Outage Probability Analysis of Co-Tier Interference in Heterogeneous Network
}

\author{
Mohammad Kamrul Hasan ${ }^{1,2}$, Ahmad Fadzil Ismail ${ }^{2}$, Wahidah Hashim ${ }^{3}$, Shayla Islam ${ }^{4}$, Aisha-Hassan \\ Abdalla Hashim ${ }^{2}$ \\ ${ }^{1}$ Department of Electrical and Electronics Engineering, University Malaysia Sarawak, \\ Kota Samarahan, 94300 Sarawak, Malaysia \\ ${ }^{2}$ Department of Electrical and Computer Engineering, International Islamic University Malaysia, \\ Jalan Gombak, Kuala Lumpur 53310, Malaysia \\ ${ }^{3}$ College of Computer Science \& Information Technology, University Tenega Nesional, \\ Malaysia \\ ${ }^{4}$ Department of Computer Science \& Engineering, Green University of Bangladesh, \\ Dhaka-1207, Bangladesh \\ hasankamrul@ieee.org
}

\begin{abstract}
In Heterogeneous Network (HetNet), the femtocell (HeNB) has been deployed by the telecommunication industries to provide extensive coverage as well as capacity in an indoor. These HeNBs are Customer Premise Equipment (CPE) which is randomly used in co-channel with macrocell (MeNB) and causes the Co-Tier Interference (CTI) in OFDMA. The effect of CTI in OFDMA systems can lead the system throughput degradation and service disruption. Because of quick direct changing features in Rayleigh channel, it is compulsory to succeed the satisfactory performance. The signal-to-interference noise ratio (SINR) is arbitrary which drives the highest capacity to be an irregular variable. However, this paper derives the expressions of outage probabilities based on the hybrid Genetic Algorithm (GA) with biogeography based dynamic subcarrier allocation (HGBBDSA) algorithm is implemented in reducing the outage probability. The outage probability countenance is expressed for the moment-generating function of the total SINR at the receivers end. The simulation results demonstrate that the HGBBDSA can lessen the outage to $45 \%$ than existing methods.
\end{abstract}

Index Terms-OFDMA; co-tier interference; HGBBDSA; heterogeneous network; LTE-A.

\section{INTRODUCTION}

Wireless operators have turned to the use of Third Generation Partnership Project (3GPP) Long Term Evolution-Advanced (LTE-A) in HetNet, in the attempt to provide better spectral efficiency, reduced delay on account of its Internet Protocol (IP) structural design, along with greater throughputs. The Cellular networks LTE/LTE-A faces the imperfect network coverage in indoor due to the multipath channel fading. To abate such challenge, HeNBs are deployed in HetNets (Fig. 1), which can easily extend the capacity up to $1.5 \mathrm{Gbps}$ in LTE-A systems and so also

Manuscript received 5 May, 2017; accepted 19 August, 2017.

This research was funded by FRGS project from the Ministry of Education, Malaysia. This research was performed in cooperation with the Universiti Tenaga Nesional Malaysia. extends the coverage up to $30 \mathrm{~m}$ in indoor houses or any high-rise multilevel buildings [1]. Generally, HeNBs are connected via third party internet broadband connectivity to the operators' network. The S2/MME interface is defined by the 3GPP2 for the HeNBs architecture [2], [3]. Strategically, the arbitrary placement of HeNBs and the spectrum sharing between MeNB highlights the significance of interference mitigation.

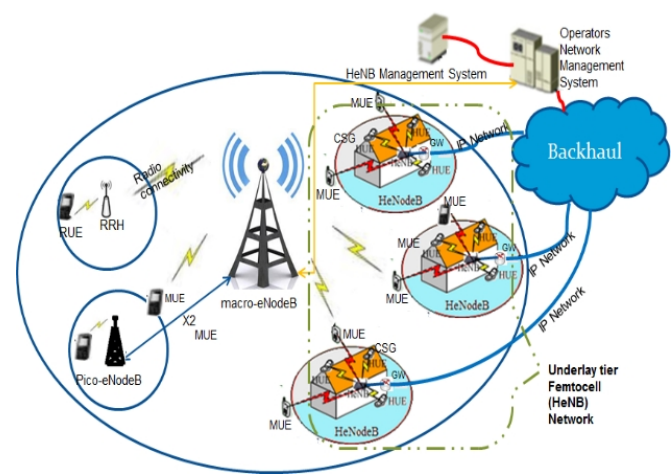

Fig. 1. Heterogeneous Network Architecture [2].

Specification of the co-channel deployment of HeNBs is to use the same spectrum as the MeNB; the consequence of closed access HeNBs has to be taken into account, during the purpose of service outage. Therefore, the outage problem has become the leading technical challenge. Due to the outage issue the HeNBs faces the lower throughput as well as higher outage. Owing to the fast channel changing features in Rayleigh radio channel, it is highly necessary to achieve the satisfactory communication performance, which is typically a certain least possible signal level [4], [5]. So, in these fading channels, the received signal depends on the channel since the power is random. In this conditions, SINR become random that leads the maximum capacity to be a random variable. Therefore, these received signals should be defined through the probability models. Considering these points. outage probability indicates the probability that a rate 
is not supported due to variable of SINR as well as User Equipment's (UEs) demand [6]. 3GPP-LTE-A is concocting frequency reuse to achieve throughput and also simplifying the radio network planning [7]. HetNets increases its capacity through aggressive Frequency Reuse (FR) technique in allocating cells smaller through HeNBs [8]. Regardless of the way that the expansion in limit activated by the accessibility of enhanced bandwidth can naturally remunerate the capacity and SINR deprivation, the capacity of HeNB User equipment's (HUEs) with low SINR [9]. The outage of HeNBs in HetNets had explored through the spatial poison point process with the consideration of lognormal fading channels [10]. To evaluate and mitigate the interference precisely the spatial point forms had been proposed [11]. Specifically, Poisson Point Process is also known as a point procedure that exactly models the situating of HeNBs in urban zone, and it has been exploited in numerous works due to the ICI management [12], [13]. However, the estimation was considered only for MeNB networks. For HeNBs, authors have given the proposals to extend the estimation up to MeNB and HeNBs with Rayleigh fading, where it has been observed that interference power is suspected [12]. The cell edge UEs experiences the higher outage and lower throughput with the expanding number of cells, which is caused by interferences [14]. Due to the effect of CTI in OFDMA, the outage rate is analysed in [6], where GA and Particle Swarm Optimization (PSO) based dynamic subcarrier allocation had been proposed.

This paper analyses the outage probability for the CTI in OFDMA based HetNets. Especially, when OFDMA resources are shared between HeNBs using the HGBBDSA algorithm. This article also presents the subcarrier allocation using the HGBBDSA algorithm for the HetNets to manage the interference in OFDMA systems. The analysis is carried out via system level simulation with the consideration of path loss, Rayleigh fading and lognormal shadowing.

The rest of the paper is structured as: System and channel models are presented in Section II. The performance of Outage Probability is discussed in Section III. Conclusions are given in Section IV.

\section{SYSTEM AND CHANNEL MODEL}

Focusing CTI scenario of HetNet in Fig. 2, presents the system model. The proposed approach applies the subcarrier detection in HeNB neighbor network.

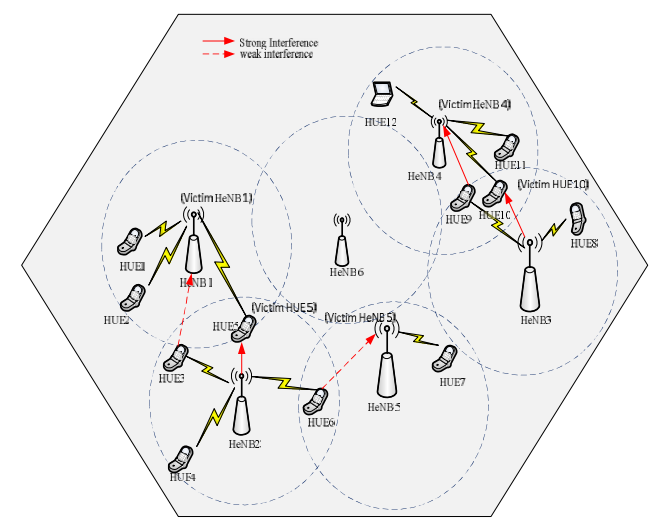

Fig. 2. Co-tier interference scenario.
The main idea is to search the unused subcarrier and then reallocate this to the HUEs. GA and $\mathrm{BBO}$ algorithm are integrated (HGBBDSA) to subcarrier search and allocation. According to the approach, the system model depicted considering MeNB and HeNB HetNet system. The overlay contains the MeNB base station as well as the MeNB User equipment's (MUEs). On the other hand, the underlay HeNB tier encompasses the installed HeNB base stations and the HUEs functioned by the HeNBs.

The HGBBDSA is accomplished the subcarrier allocation improving task that distributed inside the HeNBs and the adjacent HeNBs, by allotting the subchannel to HUE in LTE-A HetNet. HeNBs are usually deployed in a closed accessed mode by the end users and has a lack of influential backhauls, henceforth fast reacting ICIC techniques are impervious [15]. Thereby, CTI is formed by HeNBs owing to the low isolation of walls or windows. The avoidance of CTI is achievable by OFDMA HeNBs by properly assigning frequency resources among users in a bigger time scale or by self-organizing methods. In Fig. 2, the relationship of the $j^{\text {th }}$ HeNB and $i^{\text {th }}$ HUEs are concocting CTI. Thus, the SINR $\left(\gamma_{u_{h}, S}^{S I N R}\right)$ at HeNB shown as

$$
\gamma_{u_{h}, S}^{S I N R}=\frac{\Gamma_{u_{h}, S}^{j_{n}} h_{u_{h}, S}^{j_{n}} \phi_{u_{h}, S} P_{u_{h} \leftrightarrow j_{n}}}{i_{S}^{u_{h}}+t_{S}},
$$

where

$$
i_{S}^{u_{h}}=\sum_{s=1}^{S} \sum_{l=L, l \neq j_{n}}^{L}\left(\begin{array}{c}
\sum_{u_{h}, S}^{j_{n}} \Gamma_{u_{h}, S}^{j_{n}} h_{j_{n}, S}^{u_{h}} P_{u_{h} \leftrightarrow j_{n}}+ \\
\sum_{u_{h} \in l_{I_{\omega}}} \phi_{u_{h}, S}^{j l} \Gamma_{u_{h}, S}^{j_{l}} h_{u_{h}, S}^{j_{l}} P_{u_{h} \leftrightarrow j_{l}}
\end{array}\right),
$$

here $i_{S}^{u_{h}}$ stated the interference scenario, $u_{h}$ indicates the HUE, $\Gamma_{u_{h}, S}^{j_{n}}$ is the transmit power, $\phi_{u_{h}, S}$ is subcarrier allocation indicator, $h_{u_{h}, S}^{j_{n}}$ is the channel gain of HUE and serving HeNB, $t_{s}$ is the noise $P_{u_{h} \leftrightarrow j_{n}}$ is represented for indoor path loss, $j_{n}$ is the adjacent HeNB. Using Shannon's law, the capacity can be stated as

$$
\Psi_{s, i}=\sum_{s=1}^{S} \frac{\phi_{u_{h}, S}}{S} \log _{2}\left(1+\gamma_{u_{h}, S}^{S I N R}\right) .
$$

To enhance the fitness constraint, the subcarrier optimization problem is represented as:

$$
\begin{gathered}
\operatorname{MaxF}\left(\phi_{u_{h}, S}, \Gamma_{S}^{i_{n}}\right)=\sum_{l=1}^{I} \varpi_{u_{h}} \sum_{s=1}^{S}\left(\frac{\phi_{u_{h}, s}}{S} \log _{2}\left(1+\gamma_{u_{h}, S}^{S I N R}\right)\right), \\
C_{1}: \sum_{s=1}^{S} \phi_{u_{h}, s} \Gamma_{u_{h}, S} \leq \Gamma_{\text {total }}, \forall u_{h}, j_{n}, \\
C_{2}: \sum_{s=1}^{S} \phi_{u_{h}, s}=1, \forall S, \\
C_{3}: \Gamma_{u_{h}, S}=1, \forall S, u, \\
C_{4}: \phi_{u_{h}, s} \in\{0,1\}, \phi_{u_{h}, S} \leq 1, \forall s, u_{h},
\end{gathered}
$$

where total transmit power is represented as $\Gamma_{\text {total }}, \varpi_{u_{h}}$ is 
the weighting factor and the subcarrier allocation indicator which means that the maximum subcarrier is allocated, if $\left[\phi_{u_{h}, s}=1\right]$. Hence, this leads to maximum throughput for the association of HUE and HeNB.

\section{A. HGBBDSA Approach}

The procedure of the HGBBDSA algorithm is given as underneath:

1. Initially, breeds subchannels as populations. Then assigning subchannels into subcarriers.

2. A fitness function is tested whether the number of features assessment is reached or not using applying (3). The subpopulations are individually breed offspring.

3. Applies migration procedure for individual offspring to advance through the mutation. Then swap the parent with offspring subcarriers.

4. The algorithm is tried through the stopping state by testing to satisfy the fitness, generations, or dispensed time accomplishment.

5. When the stopping condition is comes across, then it saves the best solutions as the output and checks for next generation.

Applying the HGBBDSA algorithm for optimizing task into a bunch of local optimization, will lead to achieve faster convergence. A number of population (subchannel) and subpopulations (subcarrier) are generated. Where each of the populations is considered for allocation to each of local optimization. Each of the HeNB performs the optimization to compute the objective function. The total processes of offspring generation, fitness estimation, encoding, and mutations used.

\section{PERFormance ANALYSIS AND Discussion}

The performance is evaluated using Matlab based system level simulator that is enhanced with the HGBBDSA algorithm to allocate the OFDMA subcarriers. The simulation configuration has considered the 35 HUEs, 20 HeNBs in 200-meter square area. The path-loss model is deliberated as the type of links in concerning HUE and HeNB [16]. Taking in attention of CTI in between HUE and HeNB when considering the interfering link concerning the path-loss [16] valuation can be stated as

$$
\begin{gathered}
P_{u_{h} \leftrightarrow j_{n}}=38.46+20 \log _{10}(d)+ \\
+0.7 d_{2 D, \text { indoor }}+18.3 \Omega\left(\frac{(\Omega+2)}{(\Omega+1)-0.46}\right)
\end{gathered}
$$

where $d$ indicates the distance, $\Omega$ is the number of penetrated floor, $0.7 d_{2 D \text {,indoor }}$ is for indoor wall loss, $d_{2 D \text {,indoor }}$ is the distances inside the rooms for the houses of each HeNB.

Due to fast channel changing features in Rayleigh radio channel, it is highly necessary to achieve the satisfactory communication performance, which is characteristically an individual least possible signal level [4], [10]. Thus, the SINR is also random. These random SINR leads the maximum capacity to be a random variable. Hence, the Outage for HUE of $S^{\text {th }}$ subcarrier is expressed as below[5]

$$
P_{S, u_{h}}^{\text {out }}=P_{r}\left(\gamma_{u_{h, S}, S I N R}^{S I} \psi_{S, i}\right)
$$

Outage Probability is analysed as the probability SINR of HUE in a given subchannel is less than the data rates. The

\begin{tabular}{|c|c|}
\hline Parameter & Values \\
\hline Frequency & $2.6 \mathrm{GHz}$ \\
\hline HeNBs & 30 \\
\hline Bandwidth & $20 \mathrm{MHz}$ \\
\hline HeNB radius & $20 \mathrm{~m}$ \\
\hline HeNB power & $23 \mathrm{dBm}$ \\
\hline External wall loss & $10 \mathrm{~dB}$ \\
\hline Indoor wall-loss & $0 \mathrm{~dB}$ \\
\hline Noise factor & $-174 \mathrm{Bm} / \mathrm{Hz}$ \\
\hline Shadowing correlation & $0.7 \mathrm{~dB}$ \\
\hline HUEs & 40 \\
\hline Path-loss model & Suburban \\
\hline Channel model & 3GPP typical urban \\
\hline Maximum MUE transmit power & $24 \mathrm{dBm}$ \\
\hline Maximum HUE transmit power & $20 \mathrm{dBm}$ \\
\hline HeNB antenna pattern & Omni \\
\hline Population & 500 \\
\hline Generation & 500 \\
\hline Mutation factor & 0.015 \\
\hline
\end{tabular}
simulation parameters are summarized in Table I.

TABLE I. SIMULATION PARAMETERS [1], [4], [6], [16]-[19].

The evaluation of total capacity with respect to SINR in Fig. 3 suggests that, if the number of HUEs is increased then also the achieved SINR is performed better.

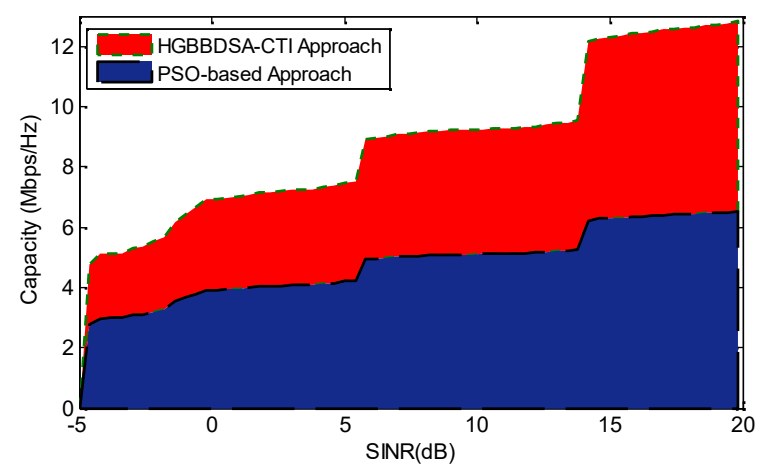

Fig. 3. Total Capacity vs. SINR.

This indicates that the subcarrier sharing with other HeNBs and the allocation to HUEs are efficient. Thus the total capacity can be extended up to $12.4 \mathrm{Mbps} / \mathrm{Hz}$ if the SINR at $20 \mathrm{~dB}$. In contrast, in low SINR $(-5 \mathrm{~dB}, 0 \mathrm{~dB}, 5 \mathrm{~dB}$ and even in $10 \mathrm{~dB}$ ) the capacity is increased to $4.5 \mathrm{Mbps} / \mathrm{Hz}$ to $9.2 \mathrm{Mbps} / \mathrm{Hz}$. Figure 3 also suggests that the existing approach achieves lower capacity compare to HGBBDSA approach.

The impact of the outage probability against quantity of HUE shown Fig. 4, where 30 HUEs are considered. The outage occurs because of the variable SINR values which may lead the most extreme ability to be arbitrary. Along these lines, the impact on the got signals and the HUEs endures by the slightest throughput. Accordingly, the lower the outage probability, the higher the imprecise (channel blurring) flag and lower the capacity. Figure 4 demonstrates 
the outage rate for 25 HUEs after the subcarrier allotment. It is clear that both of GA and PSO based approach contains the higher outage which is 0.6 if the number of HUEs is 25 than the HGBBDSA approach. The outage probability are summarized in Table II.

TABLE II. SUMMARY OF THE OUTAGE PROBABILITY RESULTS.

\begin{tabular}{|c|c|c|c|c|}
\hline & \multicolumn{4}{|c|}{ Outage probability } \\
\hline Approach details & HUE = 5 & HUE $=\mathbf{1 0}$ & HUE $=\mathbf{1 5}$ & HUE $=\mathbf{3 0}$ \\
\hline HGBBDSA-CTI & 0.09 & 0.13 & 0.22 & 0.32 \\
\hline PSO-based DSA & 0.45 & 0.47 & 0.50 & 0.65 \\
\hline GA-based DSA & 0.45 & 0.50 & 0.54 & 0.68 \\
\hline
\end{tabular}

The HGBBDSA achieves the maximum subchannel assignment outage which is around $52.9 \%$ reduced than that of GA based, and $50.76 \%$ lower than that of PSO based approach. Similarly, with the user demand, the outage rate is evaluated, and for the analysis, it is presented in Fig. 5. It can be seen from the Fig. 5 that, the HGBBDSA approach achieves lower outage rate over user demand variations. At the same note, the GA, and PSO based approaches suffer the higher outage rate.

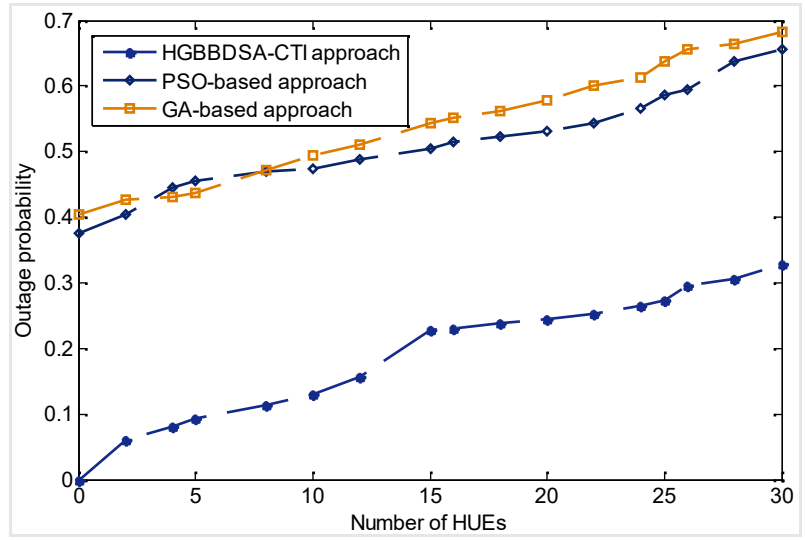

Fig. 4. Outage Probability versus Number of HUEs.

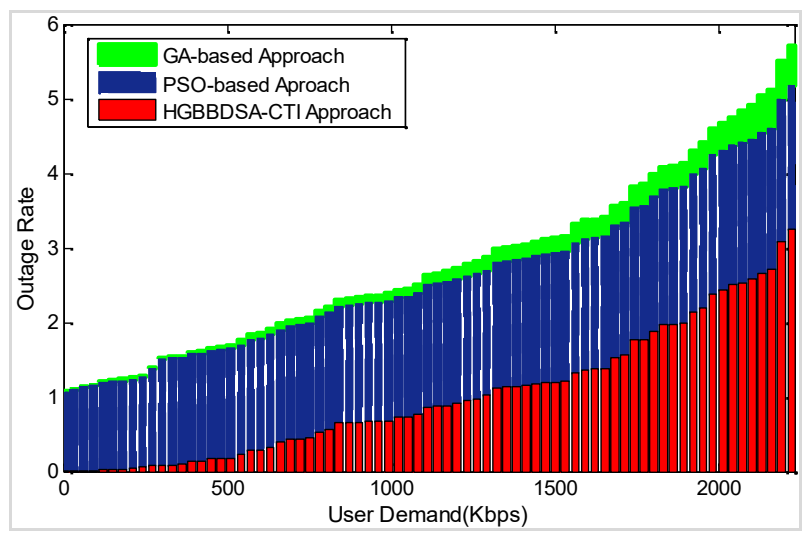

Fig. 5. Outage rate versus user demand.

The HGBBDSA approach lowers the outage since it employs a control parameter to indicate subcarrier allocation and to increase the subcarrier diversity with fitness.

\section{CONCLUSIONS}

This paper studied the performance of OFDMA system based HetNet with dynamic subcarrier allocation. The performance is mainly measured and evaluated for the HGBBDSA approach to mitigate interference in
Heterogeneous network. For this, a comprehensive analysis of the outage probability for OFDMA based HetNet is accomplished significantly. In consequence, the system and channel model have derived in order to evaluate the SINR, interference and outage rate analysis. The bio-inspired hybrid GA with the biogeography-based algorithm is used in order to search and share the subcarrier to the HeNB users dynamically. In summary, the simulation outcome recommends that the implementation of the HGBBDSA approach enhances the SINR, therefore it lowers the outage probabilities. It is also suggested that the outage rate outperforms than the existing approaches. As a result, the total capacity is increased. It is also revealed that, if the number of HUEs is increased the performance of SINR still better. This is because the HGBBDSA approach shares the available OFDMA resources with other HeNBs to allocate HUEs dynamically. Thus, the analysis recommends that if the SINR is higher so that the outage is lower which leads enhance the total capacity.

\section{REFERENCES}

[1] M. K. Hasan, A. F. Ismail, A.-H. Abdalla, K. Abdullah, H. Ramli, S. Islam, K. Badron, "Self-organizing joint sensing and power allocation scheme (SJSPA) to coordinate cross-tier interference for LTE-A heterogeneous networks", in IEEE 2nd Int. Symposium on Telecommunication Technologies (ISTT), 2014, pp. 11-16. [Online]. Available: http://dx.doi.org/10.1109/ISTT.2014.7238168

[2] M. K. Hasan, A. F. Ismail, A. H. Abdalla, H. A. M. Ramli, W. Hashim, A. Razzaque, M. H..Khairolanuar, "A self-organizing approach: time synchronization for the HeNodeBs in heterogeneous network", Advanced Computer and Communication Engineering Technology, pp. 365-374, 2016. [Online]. Available: http://dx.doi.org/10.1007/978-3-319-24584-3_30

[3] M. K. Hasan, A. F. Ismail, H. Aisha, K. Abdullah, H. Ramli, S. Islam, "Inter-cell interference coordination in Heterogeneous Network: A qualitative and quantitative analysis", in IEEE Malaysia Int. Conf. Communications (MICC), 2013, pp. 361-366. [Online]. Available: http://dx.doi.org/10.1109/MICC.2013.6805855

[4] P. P. Hasselbach, A. Klein, "An analytic model for outage probability and bandwidth demand of the downlink in packet switched cellular mobile radio networks", in IEEE Int. Conf. Communications (ICC 2008), 2008, pp. 252-256. [Online]. Available: http://dx.doi.org/10.1109/ICC.2008.54

[5] D.-C. Oh, Y.-H. Lee, "Cognitive radio based resource allocation in femto-cells", Journal of Communications and Networks, vol. 14, pp. 252-256, 2012. [Online]. Available: http://dx.doi.org/10.1109 /JCN.2012.6253085

[6] A. Shahid, S. Aslam, K.-G. Lee, "A decentralized Heuristic Approach towards resource allocation in Femtocell networks", Entropy, vol. 15, pp. 2524-2547, 2013. [Online]. Available: http://dx.doi.org/ $10.3390 / \mathrm{e} 15072524$

[7] K. Han, Y. Choi, D. Kim, M. Na, S. Choi, K. Han, "Optimization of femtocell network configuration under interference constraints", in 7th Int. Symposium on Modeling and Optimization in Mobile, Ad Hoc, and Wireless Networks, (WiOPT 2009), 2009, pp. 1-7. [Online]. Available: http://dx.doi.org/10.1109/WIOPT.2009.5291642

[8] R. Y. Chang, Z. Tao, J. Zhang, C. C. J. Kuo, "Dynamic fractional frequency reuse (D-FFR) for multicell OFDMA networks using a graph framework", Wireless Communications and Mobile Computing, vol. 13, pp. 12-27, 2013. [Online]. Available: http://dx.doi.org/10.1002/wcm.1088

[9] M. Hasan, A. F. Ismail, A. H. Abdalla, K. Abdullah, H. Ramli, $\mathrm{S}$. Islam, et al., "Inter-cell interference coordination in LTE-A HetNets: A survey on self organizing approaches", in Int. Conf. Computing, Electrical and Electronics Engineering (ICCEEE 2013), 2013, pp. 196-201. [Online]. Available: http://dx.doi.org/ 10.1109/ICCEEE.2013.6633932

[10] K. Youngju, S. Lee, D. k. Hong, "Performance analysis of two-tier femtocell networks with outage constraints", IEEE Trans. Wireless Communications, vol. 9, no. 9, pp. 2695-2700, 2010. [Online]. Available: http://dx.doi.org/10.1109/TWC.2010.070910.090251 
[11] H. Martin. Stochastic geometry for wireless networks. Cambridge University Press, 2012.

[12] H. S. Dhillon, R. Krishna Ganti, F. Baccelli, J. G. Andrews, "Modeling and analysis of K-tier downlink heterogeneous cellular networks", IEEE Journal on Selected Areas in Communications, vol. 30, no. 3, pp. 550-560, 2012. [Online]. Available: http://dx.doi.org/10.1109/JSAC.2012.120405

[13] J. G. Andrews, F. Baccelli, R. K. Ganti, "A tractable approach to coverage and rate in cellular networks", IEEE Trans. Communications, vol. 59, no. 11, pp. 3122-3134, 2011. [Online]. Available: http://dx.doi.org/10.1109/TCOMM.2011.100411.100541

[14] M. Yavuz, F. Meshkati, S. Nanda, A. Pokhariyal, N. Johnson, B. Raghothaman, et al., "Interference management and performance analysis of UMTS/HSPA+ femtocells", IEEE Communications Magazine, vol. 47, pp. 102-109, 2009. [Online]. Available: http://dx.doi.org/10.1109/MCOM.2009.5277462

[15] J. Zhang, H. Tian, P. Tian, Y. Huang, L. Gao, "Dynamic frequency reservation scheme for interference coordination in LTE-advanced heterogeneous networks", in IEEE 75th Vehicular Technology Conf.
(VTC Spring 2012), 2012, pp. 1-5. [Online]. Available: http://dx.doi.org/10.1109/VETECS.2012.6239915

[16] ITU-M.2135-1, "Guidelines for evaluation of radio interface technologies for IMT-Advanced, Series, M", Technical report, ITU, 2009.

[17] X. Kang, R. Zhang, Y.-C. Liang, H. K. Garg, "Optimal power allocation strategies for fading cognitive radio channels with primary user outage constraint", IEEE Journal on Selected Areas in Communications, vol. 29, pp. 374-383, 2011. [Online]. Available: http://dx.doi.org/10.1109/JSAC.2011.110210

[18] F. Zhou, N. C. Beaulieu, Z. Li, J. Si, P. Qi, "Energy-efficient optimal power allocation for fading cognitive radio channels: Ergodic capacity, outage capacity, and minimum-rate capacity", IEEE Trans. Wireless Communications, vol. 15, no. 4, pp. 2741-2755, 2016 [Online]. Available: http://dx.doi.org/10.1109/TWC.2015.2509069

[19] Z. Liu, Y. Yuan, L. Fu, X. Guan, "Outage performance improvement with cooperative relaying in cognitive radio networks", Peer-to-Peer Networking and Applications, vol. 10, no. 1, pp. 184-192, 2017. [Online]. Available: http://dx.doi.org/10.1007/s12083-015-0417-0 